\title{
MHD simulations of star-disk magnetospheres and the formation of outflows and jets
}

\author{
Christian Fendt \\ Max Planck Institute for Astronomy, Königstuhl 17, 69117 Heidelberg, Germany \\ email: fendt@mpia.de
}

\begin{abstract}
In this review the recent development concerning the large-scale evolution of stellar magnetospheres in interaction with the accretion disk is discussed. I put emphasis on the generation of outflows and jets from the disk and/or the star. In fact, tremendous progress has occurred over the last decade in the numerical simulation of the star-disk interaction. The role of numerical simulations is essential in this area because the processes involved are complex, strongly interrelated, and often highly time-dependent. Recent MHD simulations suggest that outflows launched from a very concentrated region tend to be un-collimated. I present preliminary results of simulations of large-scale star-disk magnetospheres loaded with matter from the stellar, resp. the disk surface demonstrating how a disk jet collimates the wind from the star and also how the stellar wind lowers the collimation degree of the disk outflow.
\end{abstract}

Keywords. Accretion disks, MHD, methods: numerical, stars: formation, stars: magnetic fields, stars: mass loss, stars: winds, outflows, ISM: jets and outflows.

\section{Introduction}

Highly collimated jets and outflows are one of the most striking signatures of young stars. There is general agreement that these jets are collimated disk/stellar winds, being launched, accelerated, and collimated by magnetic forces (see reviews by Pudritz et al. 2007; Shang et al. 2007). However, the details of the physical processes involved are not completely understood. In fact, young stellar objects may carry a strong stellar field which is important for the angular momentum exchange between disk and star (Bouvier et al. 2007), but will presumable affect the outflow formation as well.

This review discusses some of the essential aspects of outflow formation from magnetized young stars, in particular the application of numerical magnetohydrodynamic (MHD) simulations. I concentrate on the global, large-scale picture, i.e. on the question, how outflows are launched in the disk-star environment and how they probably look alike. I will not discuss details of the proposed "disk locking" mechanism in such systems, and not the issues of dipolar accretion or the evolution of the magnetized accretion disk itself. For this, I refer to the contributions by Shu, Romanova, Matt, and Ferreira.

\section{Jet formation - the standard model}

The principal processes involved in jet formation may be summarized as follows. The underlying hypothesis is that jets can only be formed in a system with a high degree of axi-symmetry (e.g. Fendt \& Zinnecker 1998).

- Magnetic field is generated by the star-disk system.

- The star-disk system also drives an electric current. 


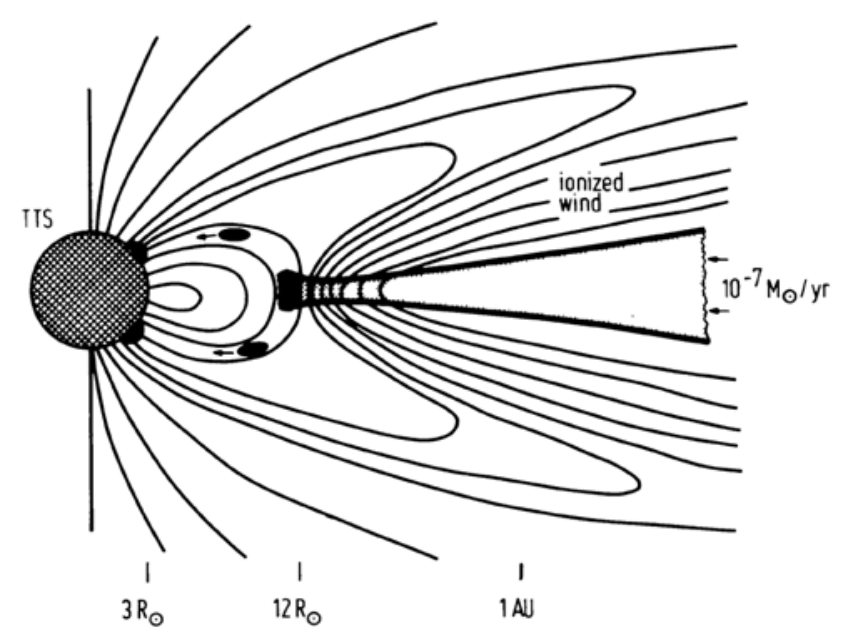

Figure 1. Magnetic star-disk interaction in young star and the generation of protostellar outflows and jets as proposed by Camenzind (1990).

- Accreting matter is launched as a plasma wind (either from the stellar or disk surface), couples to the magnetic field, and is flung out magnetocentrifugally.

- Plasma inertia leads to bending of the poloidal field (i.e. the field along the meridional plane including the jet axis).

- The plasma becomes accelerated magnetically, i.e. by conversion of Poynting flux to kinetic energy.

- Pinching forces of the induced toroidal field component eventually collimate the wind flow, forming a collimated jet structure.

- The plasma velocities subsequently exceed the speed of the magnetosonic waves. The fast magnetosonic regime is causally decoupled from outer boundary conditions.

- Where the outflow meets the interstellar medium (ISM), a bow shock develops, thermalizing the jet energy. Also, the electric current is closed via the bow shock, and the jet net current returns to the source of the current via the ISM.

Historically, we note that the model topology of dipole-plus-disk field were introduced for protostellar jet formation 25 years ago by Uchida \& Low (1981) discussing possible magnetic field configurations in such systems. Amazingly, first MHD simulations of such a configuration were performed already in the early 80's by Uchida \& Shibata (1984), Uchida \& Shibata (1985) following-up these early models. However, probably due to the success of MHD disk-jet models by Blandford \& Payne (1982), Pudritz \& Norman (1983) and the limitations (spatial and time resolution) of the early numerical simulations, this concept was somewhat repressed until the early 90's when it became evident that young stars do carry a substantial and most probably large-scale magnetic field.

To my knowledge, the first who considered the detailed physical processes involved in disk truncation and channeling the matter along the dipolar field lines in the context of protostars has been Camenzind (1990) followed by Königl (1991), Collier Cameron \& Campbell (1993), Hartmann et al. (1994) and Shu et al. (1994). This sudden boost of conforming papers from competing groups added up to the breakthrough of these ideas to the protostellar jet community. Figure 1 shows the model scenario suggested by Camenzind (1990) proposing the formation of protostellar jets from star-disk magnetospheres and including details of the star-disk interaction as angular momentum exchange ("disk 
locking"), dipolar accretion, or the turbulent diffusive boundary layer. The inner disk radius was derived as

$$
r_{\mathrm{in}}=2.4 r_{\star}\left(\frac{\alpha}{2}\right)^{\frac{2}{7}}\left(\frac{B_{\star} r_{\star}}{10^{3} \mathrm{G} 3 r_{\odot}}\right)^{\frac{4}{7}}\left(\frac{\dot{M}_{\mathrm{acc}}}{10^{-7} M_{\odot} / \mathrm{yr}}\right)^{-\frac{2}{7}}\left(\frac{h(r)}{r}\right)^{\frac{2}{7}}\left(\frac{r_{\star} c^{2}}{10^{6} \mathrm{GM}_{\star}}\right)^{\frac{1}{7}}
$$

with the disk viscosity $\alpha<1$, the disk height $h(r)$, and the stellar radius $r_{\star}$ mass $M_{\star}$, and magnetic field strength $B_{\star}$. Follow-up numerical calculations provided stationary state solutions of the magnetospheric structure and the flow dynamics on a global scale, however, still resolving the central disk-jet-star geometry (see Fendt et al. 1995; Fendt \& Camenzind 1996). The magnetic field distribution was calculated in a force-free approach and showed a rapid collimation to a cylindrical collimation. The solution could only be obtained for stellar wind type geometry, thus forming a jet from open stellar field lines. Models like this are nowadays discussed as "accretion powered stellar winds" (Matt \& Pudritz 2005; Keppens \& Goedbloed 1999; see also Matt this proceedings).

I remind of the general difficulty in solving the stationary state MHD equations. The exact solution is determined by matching a smooth transition of the flow at the (singular) critical MHD surfaces which are at an initially unknown location. All stationary state solutions published so far were derived applying essential simplifications, as self-similarity (e.g. Blandford \& Payne 1982; Sauty \& Tsinganos 1994; Li 1995; Sauty et al. 2002; Contopoulos \& Lovelace 1994; Ferreira 1997), non-global solutions (e.g. Shu et al. 1994; Pelletier \& Pudritz 1992), non local force-balance (e.g. Lovelace et al. 1991), force-free freeness (see above), or very low rotation (e.g. Sakurai 1995).

Numerical simulations of jet formation became feasible since the mid 90's and did overcome the difficulties of the stationary state approach. Of course, the disadvantage is the lack of spatial resolution and the limitation in some physical parameters like plasma-beta. In general, two approaches were made. One is prescribing the accretion disk properties as a boundary condition for the jet flow (see e.g. Ustyugova et al. 1995; Romanova et al. 1997; Ouyed \& Pudritz 1997; Krasnopolsky et al. 1999; Fendt \& Elstner 2000; Fendt \& Čemeljić 2002; Vitorino et al. 2003). The other one is to include the accretion disk structure in the numerical treatment and evolve the accretion-ejection system self-consistently (see e.g. Hayashi et al. 1996; Hirose et al. 1997; Miller \& Stone 1997; Goodson et al. 1997; Kudoh et al. 1998; Casse \& Keppens 2002; von Rekowski et al. 2003; Kuwabara et al. 2005) for either pure disk systems and/or the dipole-disk interaction.

\section{Stellar magnetosphere and large-scale outflow}

Here I discuss how the presence of a central stellar magnetic field may affect the overall jet formation process.

Additional magnetic flux. In comparison to the situation of a pure disk magnetic field, the stellar magnetic field adds substantial magnetic flux to the system. For a polar field strength $B_{0}$ and a stellar radius $R_{\star}$, the large-scale stellar dipolar field

$$
B_{\mathrm{p}, \star}(r) \simeq 40 \mathrm{G}\left(\frac{B_{0}}{1 \mathrm{kG}}\right)\left(\frac{r}{3 R_{\star}}\right)^{-3}
$$

is to be compared to the disk poloidal magnetic field provided either by a dynamo or by advection of ambient interstellar field, both limited by equipartition arguments,

$$
B_{\mathrm{p}, \text { disk }}<B_{\text {eq }}(r)=20 \mathrm{G} \alpha^{-\frac{1}{2}}\left(\frac{\dot{M}_{\mathrm{a}}}{10^{-6} M_{\odot} / \mathrm{yr}}\right)^{\frac{1}{2}}\left(\frac{M_{\star}}{M_{\odot}}\right)^{\frac{1}{4}}\left(\frac{H / r}{0.1}\right)^{-\frac{1}{2}}\left(\frac{r}{10 R_{\odot}}\right)^{-\frac{5}{4}}
$$


The stellar magnetic flux will not remain closed, but will inflate and open up as the poloidal magnetic field is sheared (e.g. Uchida \& Shibata 1984; Lovelace et al. 1995; Fendt \& Elstner 2000; Uzdensky et al. 2002; Matt \& Pudritz 2005). Some of these field lines may effectively become a disk field, and therefore follow the same processes as for disk winds. The additional Poynting flux that threads the disk may assist the jet launching by MHD forces and may serve as an additional energy reservoir for the jet kinetic energy, thus implying a greater asymptotic jet speed (Michel scaling; Michel 1969; Fendt \& Camenzind 1996).

Additional magnetic pressure. However, the stellar field also provides additional central magnetic pressure which may implicate a de-collimation of the overall outflow. The central stellar magnetic field may launch a strong stellar wind which will remove stellar angular momentum. Such an outflow will interact with the surrounding disk wind. If true, observed protostellar jets may consist of two components - the stellar wind and the disk wind, with strength depending on intrinsic (yet unknown) parameters. Note that so far this argument is "ad-hoc" and numerical simulations are needed to figure out the actual dynamical evolution (see $\S 8$ ). For a further discussion of stellar winds we refer to the contribution by S.Matt.

Angular momentum exchange by the stellar field. In the scenario of magnetic "disk locking", the stellar field which threads the disk will re-arrange the global angular momentum budget. If the star looses angular momentum to the disk (this is not yet decided by the simulations, see below), both disk accretion and outflow formation is affected. The angular momentum flow from the star is transfered by the dipolar field and is deposited close to the inner disk radius (not further out than the last closed field line). Therefore, the matter in this region may be accelerated to slightly super-Keplerian rotation which has two interesting aspects: (i) due to the super-Keplerian speed this disk material could be easily expelled into the corona by magneto-centrifugal launching (Blandford \& Payne 1982; Ferreira 1997) and form a disk wind, and (ii) the excess angular momentum will stop accretion unless it is removed by some further (unknown) process. Again, a disk outflow launched from the very inner part of the disk can be an efficient way to do this. This scenario is similar to the X-wind models (Shu et al. 1994; Ferreira et al. 2000).

The torque on the star by the accretion of disk matter is $\tau_{\text {acc }}=\dot{M}_{\text {acc }}\left(G M_{\star} r_{\text {in }}\right)^{1 / 2}($ e.g. Matt \& Pudritz 2005; Pudritz et al. 2007), with the disk accretion rate $\dot{M}_{\text {acc }}$, the stellar mass $M_{\star}$ and the disk inner radius $r_{\text {in }}$ inside the co-rotation radius. For "disk locking", the star may be braked-down by the magnetic torque due to stellar field lines connecting the star with the accretion disk outside the co-rotation radius. The differential magnetic torque acting on a disk annulus of $d r$ width is $d \tau_{\text {mag }}=r^{2} B_{\phi} B_{\mathrm{z}} d r$. However, while $B_{\mathrm{z}}$ may be derived by assuming a central dipolar field, the induction of toroidal magnetic fields (electric currents) is model dependent. This is why numerical simulations of the dipole-disk interaction that evaluate simultaneously the poloidal and toroidal field components are essential. For further discussion I refer to the contributions by Romanova and Matt.

Non-axisymmetric effects from a tipped magnetic dipole. A central dipolar field inclined to the rotation axis of star and disk may strongly disturb the axisymmetry of the system. In extreme cases this may hinder jet formation at all, while weaker nonaxisymmetric perturbation may lead to warping of the inner disk, and thus a precession of the outflow launched from this area. A rotating inclined dipole also implies a timevariation of the magnetic field which may lead to a time-variation in the mass flow rates for both the accretion disk and the outflow. 
Indeed, photometric and spectroscopic variability studies of AA Tau give evidence for time-dependent magnetospheric accretion on time scales of the order a month. MonteCarlo models of scattered light by O'Sullivan et al. (2005) were able to reproduce the observed photo-polarimetric variability which may arise by the warping of the disk being induced by a tipped magnetic dipole of $5 \mathrm{kG}$ strength.

Investigations of the warping process by Pfeiffer \& Lai (2004) using numerical simulations show that the warp could evolve into a steady state precessing rigidly. Disks can be warped by the magnetic torque that arises from the a slight misalignment between the disk and star's rotation axis (Lai 1999). This disk warping mechanism may also operate in the absence of a stellar magnetosphere as purely induced by the interaction between a large-scale magnetic field and the disk electric current and, thus, may lead to the precession of magnetic jets/outflows (Lai 2003).

Three-dimensional radiative transfer models of the magnetospheric emission line profile by Symington et al. (2005) based on the warped disk density and velocity distribution obtained by numerical MHD simulations give gross agreement with observations with a variability somewhat larger than observed (see also Harries, this proceedings).

\section{Reminder on magnetohydrodynamics}

The magnetohydrodynamic concept considers an ionized neutral fluid with averaged particle quantities as fluid quantities (e.g. mass density, current density). For example, the MHD Lorentz force $\vec{F}_{L} \sim \vec{j} \times \vec{B}$ is defined by the electric current density $\vec{j}$. In MHD, electric fields are negligible small in the rest frame of the fluid. For infinite conductivity matter is frozen into the field (or vice versa), this is the limit of ideal MHD. Resistive MHD allows for a slight slip of matter across the field.

If we consider axisymmetric flows the field components can be decomposed into a poloidal component and a toroidal component, e.g. $\vec{B}=\vec{B}_{p} \times \vec{B}_{\phi}$ The helical magnetic field lines follow (and define) magnetic flux surfaces $\Psi$ - axisymmetric surfaces of constant magnetic flux,

$$
\Psi(r, z) \equiv \frac{1}{2 \pi} \int \vec{B}_{\mathrm{p}} \cdot d \vec{A}
$$

where $d \vec{A}$ is the area element of a circular area perpendicular to the symmetry axis. With that, the Lorentz force could be de-composed into components parallel and perpendicular to the flux surfaces, $\vec{F}_{L} \equiv \vec{F}_{L, \|}+\vec{F}_{L, \perp}$ with

$$
\vec{F}_{L, \|} \equiv \vec{j}_{\perp} \times \vec{B}_{\phi} \quad \text { and } \quad \vec{F}_{L, \perp} \equiv \vec{j}_{\|} \times \vec{B}
$$

This implies that a certain configuration of electric current and magnetic field distribution may accelerate the matter along the field (parallel force component) and collimate the flow across the poloidal field (perpendicular force component). Of course, also the opposite might be true under a different field configuration - de-collimation or deceleration. Note that for both magnetic acceleration and collimation the presence of a toroidal field component is essential.

Another view of how the Lorentz force do act in jets is to rewrite the force in terms of magnetic pressure and magnetic tension applying Ampère's law, $\vec{F}_{L} \sim(\nabla \times \vec{B}) \times \vec{B}$, and the well known vector identities,

$$
\vec{F}_{L}=\nabla\left(\frac{|\vec{B}|^{2}}{8 \pi}\right)+\frac{1}{4 \pi}(\vec{B} \cdot \nabla) \vec{B} .
$$

The first term on the right hand side is the gradient of the "magnetic pressure", the second 
one represents the magnetic tension due to the field curvature. Both, magnetic pressure force and tension force may contribute to acceleration and collimation. Magnetic pressure acceleration can be the main driving force for dipolar magnetospheres if the differential rotation between the foot-points in the star and the disk will wind up the poloidal field lines, thus introducing a strong vertical toroidal pressure gradient (Uchida \& Shibata 1984; Lovelace et al. 1995). Note that a pure magnetic dipole is force-free as magnetic tension and pressure forces cancel.

What is denoted by "magneto-centrifugal acceleration" (Blandford \& Payne 1982) is in that sense not an MHD effect. What happens is that the magnetic field co-rotating with the star or the disk is so strong that it dominates the matter inertia. The magnetic field lines can be considered as "wires" with "beads" on them. For a sufficiently low inclination of the field lines, the effective potential along the field becomes instable, and any perturbation will centrifugally expel the matter along the field lines. Outflow collimation by self-generated MHD forces happens when the matter inertia becomes so strong that the magnetic field cannot dominate the matter flow any longer. Matter continues to flow under conservation of angular momentum, now dragging the field with it and bending the field lines, thus inducing a toroidal magnetic field component.

\section{Self-collimated MHD jets}

Essentially, the numerical proof of MHD jet self-collimation came by two pioneering papers investigating the time evolution of a prescribed wind launched from the accretion disk surface (Ustyugova et al. 1995; Ouyed \& Pudritz 1997). These models assume a fixed-in-time equatorial boundary condition defined by a disk in Keplerian rotation and a prescribed mass flow rate from that "disk surface" into the computational domain (the "outflow"). This allows for long-term simulations and to find potential stationary state solutions. In fact, starting from the initial condition of a magnetohydro-static equilibrium, a collimated outflow evolves, proving the stationary state models discussed before.

Further application of this approach has been done e.g. for a change of the simulation box geometry (Ustyugova et al. 1999), feedback from the outflow to the boundary condition (Krasnopolsky et al. 1999), dipolar magnetospheres (Fendt \& Elstner 2000), resistive MHD jets (Fendt \& Čemeljić 2002), time-dependent perturbations (Vitorino et al. 2003), 3D non-axisymmetric perturbations (Ouyed et al. 2003), or different disk magnetic field profiles (Pudritz et al. 2006; Fendt 2006).

However, future simulations will clearly treat the large-scale time evolution of star-jet magnetospheres including the disk evolution self-consistently. In fact, future has already begun. Simulations of jet formation including the disk evolution have been presented by Casse \& Keppens (2002), Casse \& Keppens (2004), von Rekowski \& Brandenburg (2004), Meliani et al. (2007), Zanni et al. (2007) while disk-star interaction simulations were performed by Romanova et al. (2002), Romanova et al. (2004), Küker et al. (2003). For details on the latter topic I refer to the contributions by Romanova and Ferreira.

\section{MHD simulations: stellar magnetosphere - disk interaction}

Numerical simulation of the magnetospheric star-disk interaction are technically most demanding. The nature of the object requires to treat a complex model geometry in combination with strong gradients in magnetic field strength, density and resistivity, implying a large variation in physical time scales for the three components of disk, jet, and magnetosphere, which all have to be resolved numerically. 


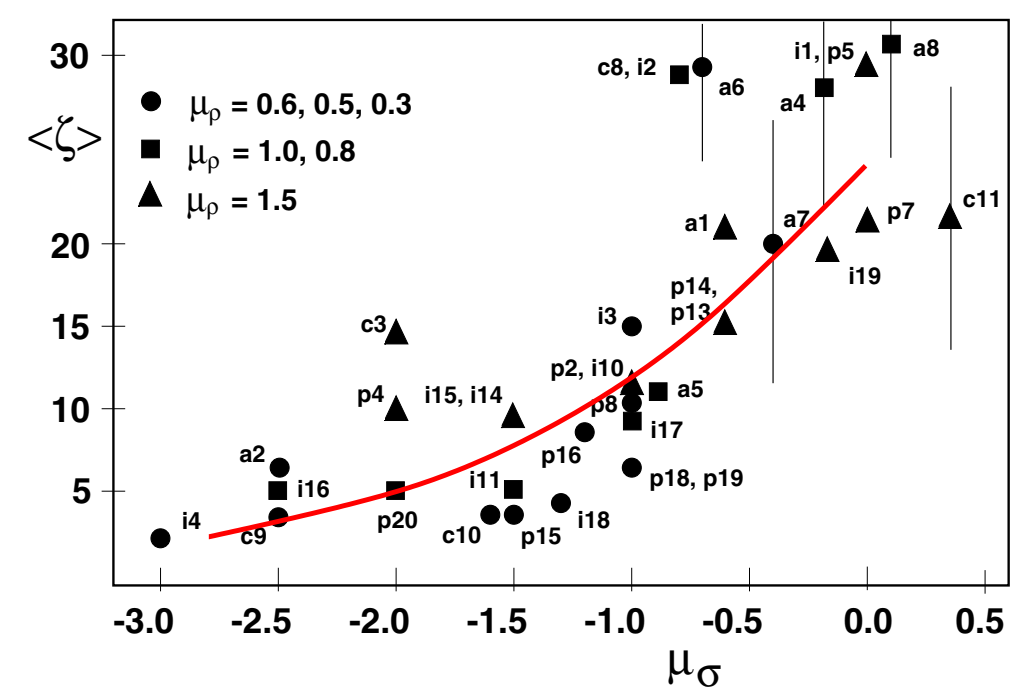

Figure 2. Collimation degree $\langle\zeta\rangle$ (averaged over differently sized regions in the computational box), plotted against the exponent of the disk wind magnetization power law profile (see Fendt 2006). The symbols trace different disk wind density profiles $\mu_{\rho}$. The different parameter runs are denoted by "a1", ..., "p20". The "error bars" for the very flat profiles indicate that here the simulation did not reach an overall quasi-steady state and, thus, the mass fluxes slightly vary in time. The line indicates the trend observed in our simulations.

Today huge progress has been made with several groups (and also codes) competing in the field. Early simulations were able to follow the evolution only for a few rotations of the inner disk (note that 100 rotations at the co-rotation radius correspond to 0.3 Keplerian rotations at 10 co-rotation radii) as e.g. Hayashi et al. (1996), Miller \& Stone (1997), Goodson et al. (1997). The next step was to increase the grid resolution and treat several hundreds of (inner) disk rotations on a global grid of $50 \mathrm{AU}$ extension (Goodson et al. 1999). The main result of these simulations is a two component flow consisting of a fast and narrow axial jet and a slow disk wind, both launched in the inner part of the disk. Close to the inner disk radius repetitive reconnection processes are seen on time scales of a couple of rotation periods. The dipolar field inflates and an expanding current sheet builds up. After field reconnection along the current sheet, the process starts all over again.

Another approach was taken by Fendt \& Elstner (2000). In order to perform long-term simulations, the evolution of the disk structure was neglected and the disk instead taken as a fixed boundary condition for the outflow. After 2000 rotations a quasi-steady state was obtained with a two-component outflow from disk and star. The outflow expands without signature of collimation on the spatial scale investigated $(20 \times 20$ inner disk radii). One result of this very long simulation is that the axial narrow jet observed in other simulations is shown to be an intermittent feature launched in the early phase of the simulation.

In a series of ideal MHD simulations Romanova and collaborators succeeded in working out a detailed and sufficiently stable numerical model of magnetospheric disk interaction. They were the first to simulate the axisymmetric funnel flow from the disk inner radius onto the stellar surface (Romanova et al. 2002$)$ on a global scale $\left(R_{\max }=50 R_{\text {in }}\right)$ and for a sufficiently long period of time in order to reach a steady state in the accretion funnel. Strong outflows have not been observed for the parameter space investigated, probably due to the matter dominated corona which does not allow for opening-up the 
dipolar field. Further progress has been achieved extending these simulations to three dimensions (Romanova et al. 2004). For the first time it has been possible to investigate the interaction of an inclined stellar dipolar magnetosphere with the surrounding disk. For further details of I refer to the contribution by Romanova in this proceedings.

The star-disk coupling by the stellar magnetosphere was also investigated by Küker et al. (2003). These simulations have been performed in axisymmetry, but an advanced disk model has been applied, taking into account $\alpha$-viscosity, a corresponding eddy magnetic diffusivity and radiative energy transport. A similar approach was undertaken by von Rekowski \& Brandenburg (2004), however allowing the disk to self-generate its own large-scale magnetic field.

\section{MHD simulations: disk jets with varying magnetic flux profile}

Here we briefly discuss recent results of numerical simulations of jet formation from disk winds (for details see Fendt 2006). A similar approach was undertaken independently by Pudritz et al. (2006). No stellar magnetic field contribution nor a stellar wind is involved, still the results have direct implication for stellar wind models. The physical grid size corresponds to $(r \times z)=(150 \times 300) r_{\text {in }}=7 \times 14 \mathrm{AU}$, which allows to resolve e.g. the velocity structure in micro jets (DG Tau).

Again we start from a force-free initial field distribution in hydrostatic equilibrium and let it evolve in time under the condition of a mass inflow from the accretion disk boundary condition. However, we extended this approach over a huge parameter run, covering a wide range of disk magnetic field profiles and disk wind mass flux profiles, both parameterized by a power law, $B_{\mathrm{p} \text {,wind }}(r) \sim r^{-\mu}, \rho_{\text {wind }}(r) \sim r^{-\mu_{\rho}}$. Both quantities could be combined in the disk wind magnetization parameter (Michel 1969), $\sigma_{\text {wind }} \sim B_{\mathrm{p}}^{2} r^{4} \Omega_{F}^{2} / \dot{M}_{\text {wind }} \sim r^{\mu_{\sigma}}$. We quantify the collimation degree by comparing the mass flux in axial and lateral direction (see Fendt \& Čemeljić 2002; Fendt 2006). Figure 2 shows the degree of collimation measured by the parameter $\zeta$ plotted against the power law exponent of the disk wind magnetization $\mu_{\sigma}$. The main result is that steep magnetization profiles (resp. disk magnetic field strength profiles) are unlikely to generate highly collimated outflows.

This important conclusion holds in particular for outflows launched as stellar winds, or X-winds, where the magnetic flux of the outflow originates in a very small region. In turn, one may say that the existence of collimated jets would require a certain disk magnetic field profile and, thus, may put some constraints on the origin of the disk, i.e. a disk dynamo-generated field versus a field advected from the interstellar medium.

\section{MHD simulations: outflows from disk-star magnetospheres}

Here I present preliminary results of MHD simulations considering a stellar magnetosphere surrounded by a disk magnetic field where both field components are fed by a mass flux from the underlying boundary condition - representing the stellar surface and the accretion disk.

The essential point is that both cases are treated, a stellar dipole aligned and antialigned with the ambient disk magnetic field. Such field geometries were considered already by Uchida \& Low (1981) and have recently been reconsidered in the form of reconnection X-winds (see contributions by S. Cabrit and J. Ferreira).

Our model setup is the following. Applying cylindrical coordinates $(r, \phi, z)$, the equatorial plane is divided in three components - the stellar surface with $r<r_{\star}=0.5 r_{\text {in }}$, the accretion disk at radii $r>r_{\text {in }}=1.0$ and the gap between star and disk. A mass 

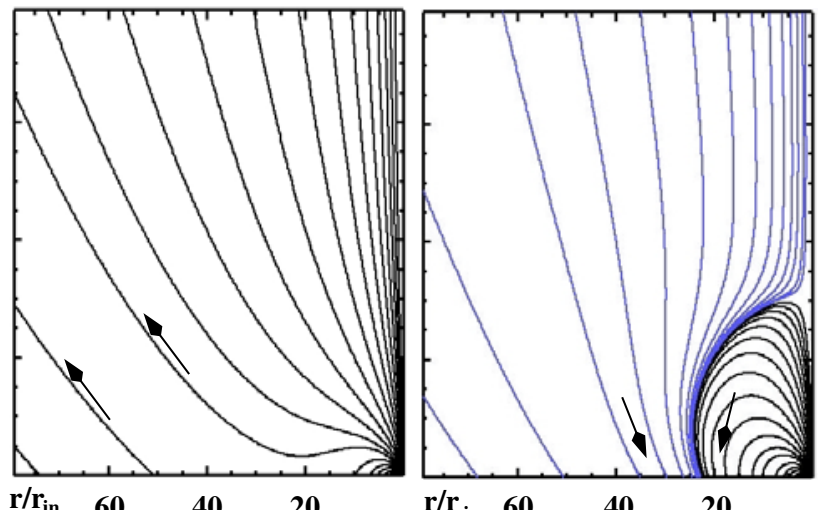

$\mathbf{r} / \mathbf{r}_{\text {in }} \mathbf{6 0} \quad 40 \quad 20$

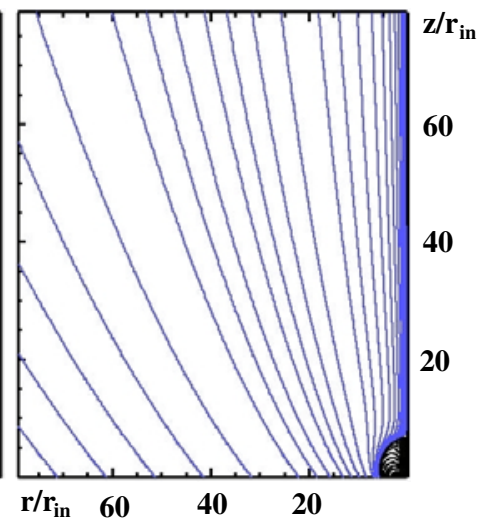

Figure 3. Initial magnetic field distribution for star-disk jet formation simulations, shown are poloidal magnetic field lines. Arrows indicate the magnetic field direction. Different strength and orientation of the superposed stellar and disk magnetic field component, $\Psi_{0, \text { disk }}=0.01,-0.01,-0.1$, resp. $\Psi_{0, \text { star }}=5.0,5.0,3.0$ (from left to right).

flux is prescribed for both wind components - star and disk. The initial magnetic field distribution is taken as a superposition of the stellar (dipolar) field and the disk field (force-free potential field),

$$
\Psi_{\text {total }}=\Psi_{0, \text { disk }} f_{\text {disk }}(r, z)+\Psi_{0, \text { star }} f_{\text {star }}(r, z)
$$

where $\Psi_{0 \text {,disk }}$ and $\Psi_{0 \text {,star }}$ measure the strength of both components and the functions $f(r, z)$ describe the initial (force-free) magnetic field distribution of both components (see Fendt 2007, to be submitted). Figure 3 shows the different initial field configurations. The magnetic flux as prescribed by the initial condition remains frozen into to the diskstar boundary. The coronal magnetic field evolves in time. The central star is rotating with a magnetospheric co-rotation radius equal to the disk inner radius. The grid size is $(r \times z)=(80 \times 80)$ inner disk radii.

Figure 4 shows how the coronal field structure evolves in time for the example simulation with $\Psi_{0, \text { disk }}=-0.1$ and $\Psi_{0 \text {,star }}=3.0$. Note that in this case the disk magnetic field and the equatorial dipolar field are aligned. This field structure has been discussed already by Uchida \& Low (1981) and is discussed nowadays in the framework of "reconnection X-winds" (see contribution by Ferreira, this proceedings). We evolve the simulations for 2800 rotations at the inner disk radius corresponding to 4 rotations at the outer disk radius. At intermediate time scales (about 700 inner disk rotations) a quasi-stationary state emerges. One clearly sees the de-collimating effect of the central stellar wind component. Note, however, that at this time the outer disk has rotated only about 0.15 times and the coronal structure above the outer disk will further evolve in time and disturb the quasi-steady state. Over the long run such quasi-stationary states may be approached again, what we observed is a cyclic behavior of the opening angle with a periodicity of about 500 (inner disk) rotation periods. We also observe that reconnection processes close to the remaining inner dipole leads to sudden flares (see also Goodson et al. 1999) which seem to trigger the large-scale cyclic behavior. The propagation of these flares is very fast, reconnection islands propagate across the jet magnetosphere within a few rotation time steps. 

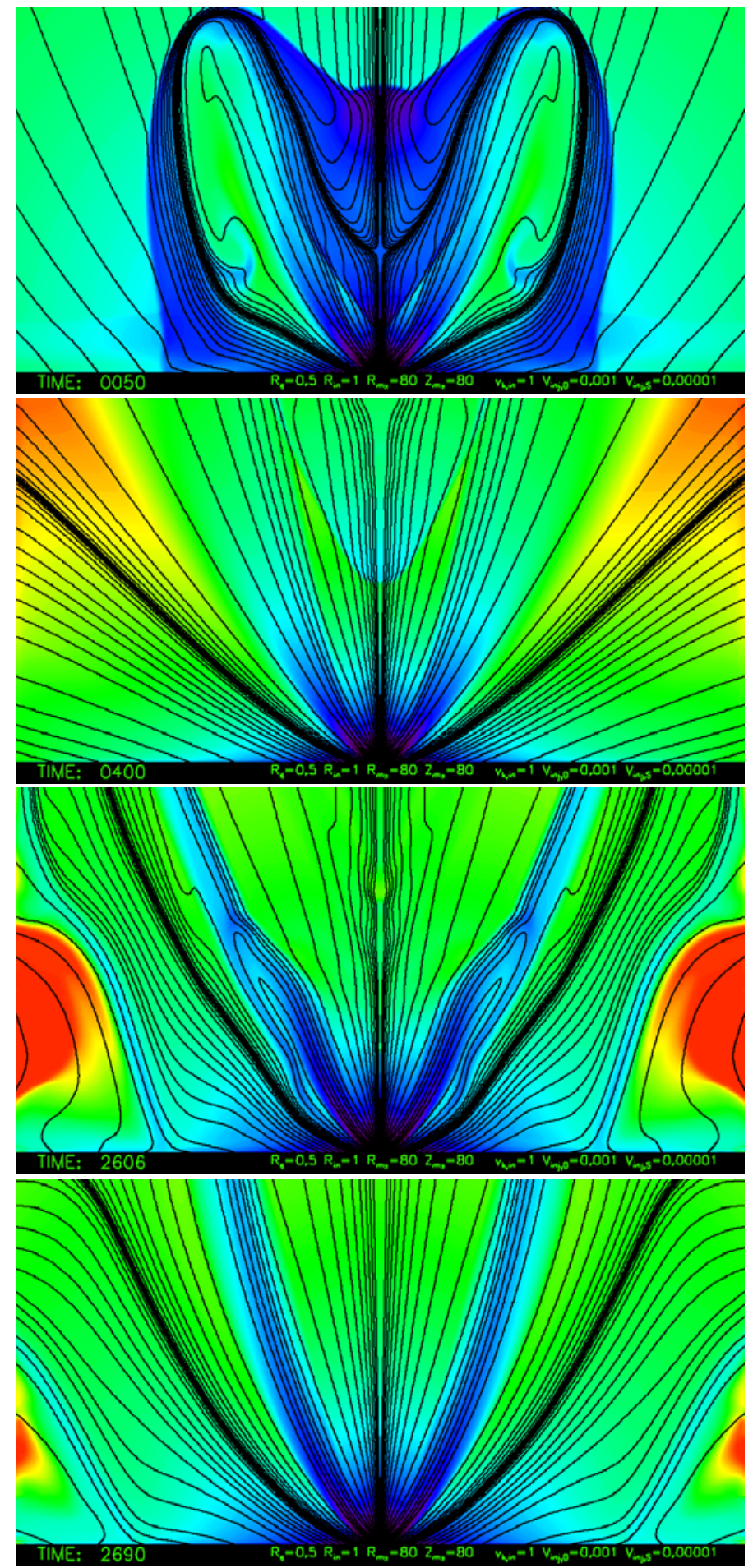

Figure 4. Time evolution of a star-disk magnetosphere from initial state of Fig. 3, right. Time step is 50, 400, 2606, 2700 rotations of the inner disk (from top to bottom). Colors show logarithmic density contours, black lines are poloidal field lines (magnetic flux contours). 


\section{Conclusions}

The stellar magnetic field has an important impact on the jet formation process. It provides an additional magnetic flux component which allows for higher outflows velocities as more magnetic energy is available to be converted into kinetic energy, and an additional central (magnetic) pressure component, which may de-collimate the outflow. It may also provide excess angular momentum in the jet launching region by disk locking. It may disturb the outflow axisymmetry, and/or trigger a time-variation in outflow rate if the central stellar magnetosphere is inclined to the rotation axis of the star-disk system. The traditional difficulties of stationary state MHD solutions have now been overcome by means of numerical simulations. Having a large number of numerical MHD codes available today, it is essential to prove the model suggestions from the past by performing numerical simulations.

Preliminary results of MHD simulations of a superposed stellar and disk magnetosphere demonstrate the de-collimation of the disk jet, respectively the collimation of the stellar wind by the surrounding disk jet. Disk jet simulations with different magnetic field and mass flux profiles provide a unique relation between disk wind magnetization and degree of collimation. Better collimation is achieved for flat magnetic field profiles. Thus, stellar winds and $\mathrm{X}$-winds are unlikely to launch highly collimated outflows.

I like to remind the reader that for a good number of codes applied in this field (as e.g. ZEUS, VAC, FLASH, or PLUTO), the numerical schemes have been published including standard tests and reference simulations. There are, however, a number of interesting publications with important and indeed convincing results, but the underlying code has never made public. Thus, it might not always be clear what the code is actually doing and what the limits of the numerical scheme are. I like both to encourage the authors of these papers to publish their codes and to advice the reader to always check the publications for their numerical background.

I conclude this review noting that tremendous progress has occurred in the numerical simulation of star-disk interaction and jet formation during the last decade. The role of numerical simulations is essential in this field because the physical processes involved are complex, strongly interrelated, and often highly time-dependent. However, it is fair to state that numerical simulations of the star-disk interaction have not yet shown the launching of a collimated jet flow comparable to the observations.

\section{References}

Blandford, R.D. \& Payne, D.G. 1982, MNRAS 199, 883

Bouvier, J., Alencar, S.H.P., Harries, T.J., Johns-Krull, C.M., \& Romanova, M.M. 2007, in: B. Reipurth, D. Jewitt, \& K. Keil (eds.), Protostars 8 Planets V, University of Arizona Press, Tucson, 2007, p.479

Camenzind, M. 1990, Reviews in Modern Astronomy 3, 234

Casse, F., \& Keppens, R. 2002, ApJ 581, 988

Casse, F., \& Keppens, R. 2004, ApJ 601, 90

Collier Cameron, A., \& Campbell, C.G. 1993, A\&A 274, 309

Contopoulos, J., \& Lovelace, R.. 1994, ApJ 429, 139

Fendt, Ch., Camenzind, M., \& Appl, S. 1995, A\&A 300, 791

Fendt, Ch., \& Camenzind, M. 1996, A $\because A$ 313, 591

Fendt, Ch., \& Zinnecker, H. 1998, A\&SA 334, 750

Fendt, Ch., \& Elstner, D. 2000, A\&SA 363, 208

Fendt, Ch., \& Čemeljić, M. 2002, A\& A 395, 1045

Fendt, Ch. 2006, ApJ 651, 272

Ferreira, J. 1997, A\&\&A 319, 340 
Ferreira, J., Pelletier, G., \& Appl, S. 2000, MNRAS 312, 387

Goodson, A.P., Winglee, R.M., \& Böhm, K-.H. 1997, ApJ 489, 199

Goodson, A.P., Boḧm, K.-H., \& Winglee, R.M. 1999, ApJ 524, 142

Hartmann, L., Hewett, R., \& Calvet, N. 1994, ApJ 426, 669

Hayashi, M.R., Shibata, K., \& Matsumoto, R. 1996, ApJ 468, L37

Hirose, S., Uchida, Y., Shibata, K., \& Matsumoto, R. 1997, PASJ 49,193

Keppens, R., \& Goedbloed, J. P. 1999, A\& A 343, 251

Königl, A. 1991, ApJ 370, L39

Krasnopolsky, R., Li, Z.-Y., \& Blandford, R.D. 1999, ApJ 526, 631

Kudoh, T., Matsumoto, R., \& Shibata, K. 1998, ApJ 508, 186

Küker, M., Henning, T., \& Rüdiger, G. 2003, ApJ 589, 397; erratum: ApJ 614, 526

Kuwabara, T., Shibata, K., Kudoh, T., \& Matsumoto, R. 2005, ApJ 621, 921

Lai, D. 1999, ApJ 524, 1030

Lai, D. 2003, $A p J$ 591, L119

Li, Z.-Y. 1995, ApJ 444, 848

Lovelace, R., Berk, H., \& Contopoulos, J. 1991, ApJ 379, 696

Lovelace, R., Romanova, M., \& Bisnovatyi-Kogan, G. 1995, MNRAS 275, 244

Matt, S., \& Pudritz, R.E. 2005, ApJ 632, L135

Meliani, Z., Casse, F., \& Sauty, C. 2007, $A \& A$ 460, 1

Michel, F.C. 1969, ApJ 158, 727

Miller, K.A., \& Stone, J.M. 1997, ApJ 489, 890

Ouyed, R., \& Pudritz, R.E. 1997, ApJ 482, 712

Ouyed, R., Clarke, D.A., \& Pudritz, R.E. 2003, ApJ 582, 292

O'Sullivan, M., Truss, M., Walker, C., Wood, K., Matthews, O., Whitney, B., \& Bjorkman, J.E. 2005, MNRAS 358, 632

Pelletier, G., \& Pudritz, R.E. 1992, ApJ 394, 117

Pfeiffer, H.P., \& Lai, D. 2004, ApJ 604, 766

Pudritz, R.E., \& Norman, C.A. 1983, ApJ 274, 677

Pudritz, R.E., Rogers, C., \& Ouyed, R. 2006, MNRAS 365, 1131

Pudritz, R.E., Ouyed, R., Fendt, Ch., Brandenburg, A. 2007, in: B. Reipurth, D. Jewitt, \& K. Keil (eds.), Protostars \& Planets V, University of Arizona Press, Tucson, 2007, p.277

von Rekowski, B., Brandenburg, A., Dobler, W., \& Shukurov, A. 2003, A\&\&A 398, 825

von Rekowski, B., \& Brandenburg, A. 2004, A\& A 420, 17

Romanova, M., Ustyugova, G., Koldoba, A., Chechetkin, V., \& Lovelace, R. 1997, ApJ 482, 708

Romanova, M., Ustyugova, G., Koldoba, A., \& Lovelace, R. 2002, ApJ 578, 420

Romanova, M., Ustyugova, G., Koldoba, A., \& Lovelace, R. 2004, ApJ 610, 920

Sakurai, T. 1985, A\&SA 152, 121

Sauty, C., \& Tsinganos, K. 1994, A\&̈A 287, 893

Sauty, C., Trussoni, E.; \& Tsinganos, K. 2002, A\& A 389, 1068

Shang, H., Li, Z.-Y., Hirano, N. 2007, in: B. Reipurth, D. Jewitt, \& K. Keil (eds.), Protostars \& Planets V, University of Arizona Press, Tucson, 2007, p.261

Shu, F., Najita, J., Ostriker, E., Wilkin, F,. Ruden, S., \& Lizano, S. 1994, ApJ 429, 781

Symington, N.H., Harries, T.J., \& Kurosawa, R. 2005, MNRAS 356, 1489

Uchida, Y., \& Low, B.C. 1981, Journal of Astroph. and Astron. 2, 405

Uchida, Y., \& Shibata, K. 1984, PASJ 36, 105

Uchida, Y., \& Shibata, K. 1985, PASJ 37, 515

Ustyugova, G., Koldoba, A., Romanova, M., Chechetkin, V., \& Lovelace, R. 1995, ApJ 439, 39

Ustyugova, G., Koldoba, A., Romanova, M., Chechetkin, V., \& Lovelace, R. 1999, ApJ 516, 221

Uzdensky, D.A., Königl, A., \& Litwin, C. 2002, ApJ 565, 1191

Vitorino, B.F., Jatenco-Pereira, V., \& Opher, R. 2003, ApJ 592, 332

Zanni, C., Ferrari, A., Rosner, R., Bodo, G., \& Massaglia, S. 2007, A\&A 469, 811 